Revista de Derecho

\title{
La legislación franquista frente al cómic femenino (1938-1977)
}

\author{
Franco's legislation against \\ the female comic (1938-1977)
}

\author{
José Joaquín Rodríguez \\ Profesor I.E.S. La Bahía - Consejería de Educación de la Junta de Andalucía, España \\ jj.rodriguez.moreno@gmail.com \\ ORCID: https://orcid.org/0000-0003-0905-8201
}

\section{Paula Sepúlveda Navarrete}

Profesora de la Universidad de Cádiz, España

paula.navarrete@uca.es

ORCID: https://orcid.org/0000-0003-1327-1320

DOI: https://doi.org/10.32719/26312484.2020.33.6

Fecha de recepción: 1 de marzo de 2018

Fecha de aceptación: 17 de diciembre de 2018

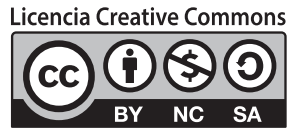




\section{RESUMEN}

La dictadura del general Franco impuso a las publicaciones españolas dirigidas a la infancia y la juventud un estricto control mediante el uso de la censura, la cual dio lugar a una limitación importante en lo que el personal creativo y las editoriales podían mostrar en los cómics y revistas que publicaban. El presente artículo estudia, a través de la legislación recogida en el Boletín Oficial del Estado, entrevistas y diferentes textos teóricos publicados durante la dictadura, quiénes eran las personas encargadas de crear y supervisar la normativa censora, y qué objetivos se marcaban en el caso de las publicaciones para mujeres jóvenes.

PALABRAS CLAVE: franquismo, censura, historieta española, estudios de género.

\section{ABSTRACT}

General Franco's dictatorship imposed publications for children and teenagers a strict censorship system, which generated an important limitation for authors and editors of comic books. This article analyses -through the legislation of the Boletín Oficial del Estado, interviews and theoretical texts published during the dictatorship- who were the people in charge of creating the regulations and supervising the contents, and what objective they were looking after in the case of female teen comics, which were publications aimed at female readers.

KeYwords: Francoism, Censorship, Spanish Comics, Gender Studies.

\section{INTRODUCCIÓN}

$\mathrm{L}$ a dictadura del general Francisco Franco (1939-1975) supone un punto y aparte en la historia de España, al romper con la tradición de corte liberal y poner fin a la experiencia democrática para, en su lugar, imponer los valores de autoridad y jerarquía. Esto se va a traducir en una transformación de la sociedad, pero no solo desde un punto de vista de clase, sino también de género, creándose un nuevo marco jurídico y social para las mujeres, que pasarán a adoptar un papel secundario y subordinado a los hombres. ${ }^{1}$

1. Pilar Domínguez Prats y María del Carmen García-Nieto París, "Franquimo: represión y letargo de la conciencia feminista, 1939-1977”, en Historia de las mujeres. Una historia propia, ed. Bonnie S. Anderson y Judith P. Zinsser (Barcelona: Crítica, 1991), 641-3. 
Para justificar esta subordinación al varón se recurre al catolicismo y a la imposición de los valores y las costumbres de corte más conservador, ${ }^{2}$ que llegan a adquirir un carácter que Román Gubern no duda en tildar de integrista. ${ }^{3}$ Dichos valores y creencias se introducen a través de la educación, que en el caso de las mujeres va a tener lugar en tres niveles diferentes y al mismo tiempo complementarios.

El primer nivel es el de la educación primaria y secundaria, controlada por la Iglesia, ${ }^{4}$ y que resulta en una escuela segregada por sexos, la existencia de asignaturas como enseñanza del hogar y exámenes obligatorios de temas domésticos para las jóvenes que aspiren a acceder a una educación superior. ${ }^{5}$ Seguidamente tenemos la Sección Femenina, la rama femenina de la Falange Española Tradicional y de las JONS (Juntas de Ofensiva Nacional Sindicalista), el partido único existente durante el Franquismo, que va a obligar a las jóvenes españolas a realizar una labor de servicio social de seis meses (si no se cumplimenta este servicio, no se puede obtener un título académico ni pasaporte, ni carnet de conducir, siendo además requisito imprescindible para trabajar como funcionaria). ${ }^{6}$ Finalmente, el adoctrinamiento de la población femenina se refuerza mediante los medios de masas, cuyo mensaje el Estado va a controlar tanto de forma directa (mediante la gestión directa de emisoras de radio, televisión, periódicos, etc.) como indirecta (mediante la creación de una legislación censora, las subvenciones públicas, etc.). ${ }^{7}$

De hecho, la prensa adulta (periódicos, revistas, panfletos, etc.) se ve desde un primer momento como una herramienta para adoctrinar a la población, y ya la primera legislación sobre prensa de la dictadura deja claro que la letra escrita es "órgano decisivo en la formación de la cultura popular y, sobre todo, en la creación de la conciencia colectiva". ${ }^{8}$ Pero además de esta prensa para adultos, también hallamos las revistas infantiles y juveniles (generalmente llamadas tebeos o cómics, si bien no están compuestas exclusivamente por historietas, sino que además ofrecen al público relatos, apartados de amistad por correspondencia, artículos, etc.), que resultan una

2. José Sánchez Jiménez, "La jerarquía eclesiástica y el Estado franquista: las prestaciones mutuas", Ayer 33 (1999): 169.

3. Román Gubern, La censura. Función política y ordenamiento jurídico bajo el franquismo (1936-1975) (Barcelona: Península, 1981), 21-2.

4. Santos Juliá, "La sociedad", en Franquismo: El juicio de la historia, coord. J. L. García Delgado (Madrid: Temas de Hoy, 2005), 101-2.

5. Pilar Folguera Crespo, "El Franquismo. El retorno a la esfera privada (1939-1975)", en Historia de las Mujeres en España, ed. Elisa Garrido (Madrid: Síntesis, 1997), 537.

6. Ibíd., 545.

7. Juan P. Fusi, "La cultura", en Franquismo: El juicio de la historia, 225.

8. España, Ley de prensa, de 23 de abril, Boletín Oficial del Estado 550, 24 de abril de 1938, preámbulo. 
forma de diversión muy popular durante buena parte de la dictadura. ${ }^{9}$ Es por ello que resulta lógico que pronto apareciesen personas interesadas en transformar este medio en una herramienta para llegar a la población más joven, transformando lo que era una vía de escape de la realidad en un sistema de adoctrinamiento; un ejemplo de ello lo tenemos en fray Justo Pérez de Urbel, que en su papel como director de la revista juvenil Flechas y Pelayos, intenta convertir la publicación en "complemento de la escuela [que] debe también tener como finalidad primaria la formación [...] religiosa, moral, patriótica, científica y humana". ${ }^{10}$

Desde el Estado se va a asegurar que este adoctrinamiento se realice gracias al control ejercido por la censura, desarrollada en un primer momento a través de una legislación general, y concretada a partir de los años 50 en leyes específicas para las publicaciones infantiles y juveniles. Dicha legislación se ha estudiado para el caso de las revistas de cómics generalistas y masculinas, ${ }^{11}$ pero tan solo ha sido tratada de pasada en el caso de las publicaciones femeninas. Sin embargo, el propio hecho de que existiera una segregación de las revistas dependiendo del sexo a quien fuesen dirigidas ya nos indica que las ideadas para las niñas y las adolescentes iban a poseer características particulares.

Obviamente, uno o varios tebeos no pueden imponer un modelo de mujer, igual que tampoco lo puede conseguir una película o una novela; no obstante, cuando existe un discurso sistemático enviado desde las aulas, la prensa, la radio y los altares, un tebeo sirve para reafirmar esos límites y para reconducir las expectativas de las lectoras. Por lo tanto, la legislación que se generó durante la dictadura sobre las publicaciones infantiles y juveniles no puede entenderse solamente como una curiosidad o una anécdota, sino como una forma de entender mejor el concepto de mujer que el régimen del general Franco deseaba imponer.

\section{METODOLOGÍA}

En su intento de moldear mediante la ley las obras culturales y artísticas, la dictadura busca mostrar unos modelos acordes a sus propios intereses; de este modo, la representación deja de ser una mera cuestión artística para convertirse en un tema

9. Antonio Martín, Apuntes para una historia de los tebeos (Barcelona: Glénat, 2000), 110-1.

10. Justo Pérez de Urbel, "Las revistas infantiles y su poder educador", Revista nacional de educación n. ${ }^{\circ} 1$ (1941): 56.

11. Por ejemplo, en Ignacio Fernández Sarasola, La legislación sobre historieta en España (Sevilla: AcyT Ediciones, 2014), y Vicent Sanchís, Tebeos mutilados: la censura franquista contra Editorial Bruguera (Barcelona: Ediciones B, 2010). 
político. ${ }^{12}$ Por lo tanto, nuestro objetivo en las siguientes páginas va a ser comprender los límites impuestos por la legislación, conocer a las personas que creaban dichas normas y ver hasta qué punto eran realmente aplicadas.

Para conseguirlo, vamos a recurrir por supuesto a la propia legislación creada durante la dictadura, principalmente a las dos Leyes de Prensa de la dictadura (1938 y 1966), la Orden que regula los contenidos para publicaciones infantiles y juveniles (1955) y el Decreto que modifica dicha regulación (1967). Todos estos textos aperecieron publicados en el Boletín Oficial del Estado y eran por lo tanto, de acceso público.

Ahora bien, ¿es suficiente la legislación? Obviamente no, pues en ocasiones no basta solo con conocer la norma, sino también el espíritu que la inspiró. Es por ello que hemos consultado también la normativa relativa a los dos órganos que asesoraron al gobierno en esta materia: la Junta Asesora de la Prensa Infantil (JAPI) entre 19521962 y la Comisión de Información y Publicaciones Infantiles y Juveniles (CIPIJ) de 1962-1977. De este modo pretendemos descubrir los perfiles de las personas que se encargaban tanto de crear las leyes como de supervisarlas.

Finalmente hemos de recordar que la ley, por el mero hecho de existir, no acarrea su cumplimiento. Se necesitan recursos materiales y humanos para que tenga un efecto real, e incluso cuando dichos medios están disponibles nos encontramos con la posibilidad de que las normas se apliquen con matices y sesgos. Por ello, para conocer el modo en que la legislación censora franquista se aplicó e influyó sobre los cómics femeninos hemos recurrido a los testimonios de artistas y editores que vivieron de primera mano su aplicación.

\section{LA PRIMERA NORMATIVA REGULADORA (1938-1951)}

La censura de las publicaciones infantiles y juveniles conoce en la España Franquista tres períodos. El primero puede considerarse una etapa de censura general, en el que no hay un corpus legislativo específico, empleándose las mismas normas censoras para todos los medios escritos independientemente de la edad y sexo de sus receptores. Esta normativa general no es otra que la Ley de Prensa de 1938, que obliga a todas las publicaciones a pasar por la revisión de los encargados de la censura antes de llegar a

12. Margaret Marshment, "The Picture is Political: Representation of Women in Contemporary Popular Culture", en Introducing Women's Studies, ed. Victoria Robinson y Diane Richardson (Londres: MacMillan Press, 1997), 125. 
los puntos de venta. ${ }^{13}$ El texto es obra de Ramón Serrano Súñer, cabeza en ese momento del Ministerio de Interior, cuñado del dictador y uno de los principales arquitectos del Estado franquista. ${ }^{14}$ La responsabilidad de vigilar los contenidos de la prensa recae principalmente sobre funcionarios públicos, aunque entre 1941-1946 se encargará el partido único del régimen, Falange Española Tradicionalista y de las JONS. ${ }^{15}$

Parece que las autoridades del régimen no sintieron especial preocupación por las revistas dirigidas a los menores, pues a fin de cuentas la situación del mercado editorial tras la Guerra Civil era crítica, sobreviviendo apenas unas pocas publicaciones destinadas al público joven, que además pertenecían en su mayoría al régimen, ${ }^{16}$ que ya durante los años de contienda se había preocupado en poner al frente de las mismas a personas cercanas a la dictadura. Por ejemplo, al frente de Flechas y Pelayos se hallaba un religioso, fray Justo Pérez de Urbel, estrechamente vinculado a la Sección Femenina de la Falange; mientras que Consuelo Gil Roësset, conocida por su papel en la prensa sublevada durante los años de guerra, iba a ser la encargada de dirigir Chicos y Mis Chicas. Será esta última la que señale la importancia de contar con una persona que dirija la dirección en sintonía con los valores del régimen: "El contenido de la publicación es lo de menos; lo que hace falta revisar es el contenido del director: su escrupulosidad, su conocimiento de la infancia, los móviles que le llevan hacia el niño". ${ }^{17}$

Por ello, aunque bien es cierto que en los años 40 parece haber una dejadez legislativa por parte de la dictadura hacia estas publicaciones, lo cierto es que sí las tenía en cuenta, solo que en lugar de crear un marco legal para un puñado de cabeceras, se había considerado más oportuno poner al frente de las mismas a personas adeptas al régimen. Habrá que esperar a los años 50, cuando con la mejora de la economía empiezan a proliferar nuevas publicaciones con contenido más centrado en el entretenimiento, para que el régimen se plantee crear una legislación que permita el control de unas revistas que poco a poco empiezan a superar en número y público a las publicaciones oficialistas. ${ }^{18}$

En este primer momento las normas reguladoras son tremendamente vagas, hasta el punto de que la ley no explica en momento alguno qué es lo que se puede o no

13. España, Ley de prensa, de 23 de abril.

14. Paul Preston, Franco. Caudillo de España (Barcelona: DeBolsillo, 2002), 287.

15. Fernández Sarasola, La legislación sobre historieta en España, 22-3 y 60-3.

16. Manuel Barrero, "Nueva mirada sobre la producción editorial de tebeos durante los años cuarenta", Espacio, tiempo y forma. Serie V, n. ${ }^{\circ} 26$ (2014): 96.

17. Consuelo Gil Roësset, La Pedagogía en la prensa infantil: conferencia pronunciada por Consuelo Gil Roësset de Franco el día 22 de mayo de 1947 (Madrid: Escuela Social de Madrid, 1947), 3.

18. Martín, Apuntes para una historia de los tebeos, 118. 
publicar. Más que un error o un vacío, nos encontramos ante una estrategia del régimen para hacer el control más efectivo, pues en lugar de limitar a los encargados de la censura con una rígida normativa en la que las empresas editoras puedan encontrar huecos a los que aferrarse, se prefiere confiar en el criterio de las personas al frente de la censura, lo que además sirve para que las editoriales no terminen de tener claros dónde están los límites, obligándolas a un ejercicio de autocensura. ${ }^{19}$

Por lo tanto, la dictadura no busca simplemente prohibir ciertos temas, sino que desea reflejar ciertos valores. ${ }^{20} \mathrm{Y}$ puesto que los valores en los que se quiere adoctrinar a las jóvenes no son siempre idénticos a los que se lanzan a los jóvenes, se van a segregar las publicaciones por sexos. En realidad, la ley no exige hasta 1955 que las publicaciones para adolescentes estén divididas por sexos, ${ }^{21}$ pero en la práctica sí se exige que se identifique claramente al público, de tal manera que algunas de las cabeceras más populares de los años 40 y primera mitad de los 50 indican quiénes son las receptoras: Mis Chicas (iniciada en 1941) no solo deja claro su público con su nombre, sino también en el anuncio de portada de que la publicación sale al mercado con censura eclesiástica para niñas mayores; Florita (1949) y Lupita (1950) señalan ambas en su título que son revistas para niñas; Ardillitas (1950) indica que contiene cómics para niñas, y Mariló (1950) explica que es una publicación ideal para niñas. Otras colecciones aparentemente no indican sexo, pero sus títulos se refieren a nombres propios femeninos, lo que sin duda orienta al público: Azucena (1946), Margarita (1951), etc. El hecho de que las publicaciones se segreguen debemos entenderlo como un intento de mandar mensajes concretos a cada sexo: lo que se explica a las lectoras no está destinado a los lectores, y viceversa.

¿Y cuáles son esos valores femeninos que la dictadura propone? Entre ellos encontramos los relacionados con el ideal de femineidad, que representan a las mujeres como personas alejadas de la razón, más sujetas a las emociones, los sentimientos y la religiosidad. ${ }^{22}$ Se las concibe de este modo como complementos indispensables de los hombres: si ellos poseen la razón que les permite desenvolverse en el espacio público, ellas han nacido para desenvolverse en la esfera doméstica como madres y esposas, ${ }^{23}$ roles que pueden desempeñar perfectamente gracias a sus capacidades emocionales

19. Fernández Sarasola, La legislación sobre historieta en España, 32-3.

20. Ibíd., 135-6.

21. España, Orden de 24 de junio de 1955, por la que se desarrolla el Decreto sobre ordenación de las publicaciones infantiles y juveniles, Boletín Oficial del Estado 33, 2 de febrero de 1956, art. 6.

22. Inmaculada Blasco Herranz, "Más poderoso que el amor: género, familia, piedad y política en el movimiento católico español", Pasado y Memoria. Revista de Historia Contemporánea, n. ${ }^{\circ} 7$ (2008): 84.

23. Aurora Morcillo Gómez, En cuerpo y alma. Ser mujer en tiempos de Franco (Madrid: Siglo XXI, 2015), $68-9$. 
y sus habilidades de cuidadoras. ${ }^{24}$ Un buen ejemplo de cómo esta forma de control funciona lo hallamos en Mis Chicas, la revista para niñas más importante de los años 40 , en donde las lectoras se encuentran secciones dedicadas al cultivo de las cualidades morales y la preparación para el matrimonio y la maternidad, convirtiéndose sus páginas en "un vehículo perfecto para la transmisión de los valores que debían tener las niñas". 25

Sin embargo, esta forma de control basada en los directores y directoras de las publicaciones va a comenzar a resultar poco eficaz ante el desarrollo de la industria editorial y la consecuente multiplicación de editoriales y publicaciones, dando lugar en los años 50 a los primeros proyectos para crear una legislación específica y detallada.

\section{LOS INTENTOS DE REGULAR LAS PUBLICACIONES INFANTILES Y JUVENILES (1952-1962)}

La mejora de la situación económica de España a partir de los años 50 favorece una mayor oferta de títulos infantiles y juveniles por parte de editoriales privadas que escapan al control directo del Estado. La propia legislación reconoce que, debido a la proliferación de estas revistas, es necesario reforzar el control sobre ellas. ${ }^{26} \mathrm{La}$ tarea cae en manos del Ministerio de Información y Turismo, encargado de los medios de comunicación del país, que en 1952 reune una mesa de expertos asesores, la JAPI. La justificación para ello es que la lectura de revistas infantiles y juveniles "puede influir de modo considerable en su formación". ${ }^{27}$

La JAPI se compone en un primer momento de ocho miembros que no dejan duda de su simpatía por los valores franquistas: destacan los ya mencionados directores de Flechas y Pelayos y de Mis Chicas. También hallamos personas de fuertes convicciones católicas, como Carmen Enríquez de Salamanca Díez, presidenta de la Asociación de las Jóvenes de Acción Católica, y José María Hueso Ballester, secretario de la Confederación Nacional de Padres de Familia. Finalmente, encontramos a personas vinculadas a Falange y sus publicaciones, como Antonio Casas Fortuny, Elisa de Lara

24. Celia Valencia Fernández, "Las políticas para las mujeres trabajadoras durante el franquismo", en Mujeres y hombres en la España franquista: Sociedad, economía, política, cultura, ed. Gloria Nielfa Cristobal (Madrid: Editorial Complutense, 2003), 162-3.

25. María del Pilar Loranca de Castro, "Mis Chicas y su influencia en las niñas de posguerras", Historietas n. ${ }^{\circ}$ 3 (2013): 74.

26. España, Decreto de 24 de junio de 1955, por el que se establecen las normas a que han de ajustarse las publicaciones infantiles y juveniles, preámbulo.

27. España, Orden de 21 de enero de 1952 por la que se crea la Junta Asesora de la Prensa Infantil, Boletín Oficial del Estado 21, 1 de febrero de 1952, preámbulo. 
Osío, Juan Antonio de Laiglesia González y Félix Valencia y Pérez de Ayala. ${ }^{28}$ No hay duda posible de la vinculación de estas personas con los valores del régimen.

La JAPI es la encargada de crear en 1955 las primeras normas reguladoras (camufladas bajo el eufemismo de orientadora) dirigidas concretamente a las publicaciones infantiles y juveniles. El objetivo de la misma queda claro desde un primer momento, pues las nuevas reglas se justifican en "una ordenación legal que garantice la recta orientación religiosa, moral, política y cultural de las [publicaciones infantiles y juveniles]". ${ }^{29}$ Además, las funciones de la JAPI son bastante amplias: deben velar por el cumplimiento de las normas, revisar si las nuevas publicaciones son adecuadas y solicitar cambios en los contenidos de las que ya se están publicando.$^{30}$ Este último punto resulta especialmente importante porque, aunque en ningún lado se especifique que las normas deban aplicarse a revistas para público masculino o femenino, se hace evidente que algunas van a tener un peso mayor en las lecturas femeninas, donde es habitual que se muestren temas relacionados con la familia y el amor, ${ }^{31}$ encontramos de esta manera la prohibición de hacer cualquier referencia a infidelidades conyugales, divorcios, situaciones que puedan excitar morbosamente o despertar la curiosidad malsana en orden a la fisiología de la generación, burlas hacia la institución familiar y las costumbres españolas y mención a romances que no se traten de manera idílica. ${ }^{32}$ Pese a ser más específica, la legislación sigue mostrando cierto nivel de ambigüedad, como al prohibir los amores ilegítimos, sin que quede claro a qué se refiere: ¿puede referirse a una relación extramatrimonial o simplemente a una en la que los jóvenes no posean la autorización de los padres?, ¿entrarían en ellos, debido al caracter ultraconservador de la dictadura, una pareja interracial o un romance en el que la mujer fuera mucho mayor que el hombre? Las editoriales debían arriesgarse y esperar al dictado de la censura.

Sin embargo, y a pesar de la legislación existente, el editor e investigador Antonio Martín señala que la JAPI "no fue operativa de forma real y práctica [...] de forma continua, organizada y regular, debido a la falta de una estructura y un organigrama oficiales" ${ }^{33}$ Es decir, que la Junta no cuenta con los recursos ni canales para hacer

28. España, Orden de 5 de febrero 1952 por la que se crea la Junta Asesora de la Prensa Infantil, Boletín Oficial del Estado 52, 21 de febrero de 1952.

29. Boletín Oficial del Estado (2 febrero 1956).

30. España, Decreto 2551/1962 de 27 de septiembre, por el que se reorganiza la Dirección General de Prensa, Boletín Oficial del Estado 245, 12 de octubre de 1962, arts. 14-19.

31. Juan Antonio Ramírez, El “comic” femenino en España. Arte sub y anulación (Madrid: Cuadernos para el Diálogo, 1975), 164.

32. España, Orden de 24 de junio de 1955, por la que se desarrolla el Decreto sobre ordenación de las publicaciones infantiles y juveniles, arts. 14-18.

33. Antonio Martín, entrevista realizada por los autores vía email entre los días 21 y 25 de julio de 2017. 
llegar sus directrices a los censores, que siguen por lo tanto teniendo una gran libertad a la hora de censurar las publicaciones que llegan a sus manos. Por ejemplo, el editor Manel Domínguez Navarro indica que el encargado de la censura en Barcelona revisa el material que la editorial le entraga de forma inmediata, sin plantearse los criterios de la JAPI: "Se le suministraban pruebas de grabador, y en ellas miraba lo que más les interesaba: escotes, faldas, curvas insinuantes, etc." ${ }^{34}$ Por los diversos testimonios parece que los censores se preocupan más por las imágenes que por los contenidos indicados en la normativa reguladora, pidiendo cambios tan subjetivos como alterar los peinados de algunos personajes de humor por considerarlos eróticos. ${ }^{35}$

Pese a su arbitrariedad, las editoriales aceptan este control sin mayores problemas, tomando de hecho la costumbre de autocensurarse ellas mismas y mostrando sin mayores problemas un mensaje conservador en lo tocante a las publicaciones femeninas. Por ejemplo, el dibujante Francisco Ibáñez, creador de los personajes Mortadelo y Filemón, recuerda: "Yo dibujaba con un ojo puesto en la página y otro en la censura, para curarme en salud". ${ }^{36}$ Jiménez Morales señala en su investigación sobre Florita, una de las publicaciones juveniles femeninas más relevantes de los años 50, que los contenidos tienen un carácter aleccionador del que carecen las publicaciones dirigidas a los chicos, planteando a las lectoras que su vida debía ser un constante perfeccionamiento para cumplir mejor con sus deberes familiares y domésticos. ${ }^{37}$ Por lo tanto, pese a la existencia de editoriales independientes y a la escasa aplicación de la legislación específica, las publicaciones femeninas continúan mostrando unos roles femeninos muy concretos y limitados, si bien es cierto que dejan de lado los elementos más adoctrinadores, como son la instrucción religiosa. ${ }^{38}$

Esta sumisión ante la censura, en la que editoriales y personal creativo se amoldaron sin mayor problema a las normas que el régimen les imponía, debe buscarse no tanto en una sintonía ideológica como en el modelo económico de las editoriales de revistas infantiles y juveniles. Y es que, como señala Terenci Moix, la industria del tebeo español se había especializado en la venta de un producto barato, cuyos beneficios se hacían palpables solamente cuando se producía y vendía en abundancia..$^{39}$ Esto

34. Manel Domínguez Navarro, entrevistado en Tebeosfera en 2010, 〈http://www.tebeosfera.com/documentos /textos/manel_dominguez_navarro_entrevista.html〉, consulta 9 de marzo de 2016.

35. Fernández Sarasola, La legislación sobre historieta en España, 147.

36. Francisco Ibáñez, entrevistado en El Mundo en 2017, 〈https://www.elmundo.es/papel/cultura/2017/11/17 /5a0db5bfe2704ea07e8b45ad.html, consulta 10 de mayo de 2019.

37. Rosario Jiménez Morales, "Pequeños defectos que debemos corregir: aprendiendo a ser mujer en la historieta sentimental de los años cincuenta y sesenta", ARBOR, vol. CLXXXVII, extra 2 (2011): 161.

38. José J. Rodríguez Moreno, "La imposición de los valores católicos patriarcales a través de la censura en las revistas juveniles femeninas de la España franquista (1941-1977)", Feminismo/s n. ${ }^{\circ} 28$ (2016): 246.

39. Terenci Moix, Historia social del cómic (Barcelona: Ediciones B, 2007 [1968]), 162-3. 
quiere decir que el personal artístico no podía permitirse el riesgo de perder varios días de trabajo por tener que repetir una o varias páginas que no gustasen a la censura (recordemos que los retoques de páginas no se pagaban), lo que ayuda a explicar el cuidado que Ibáñez ponía en no sobrepasarse lo más mínimo; además, las editoriales pedían una producción constante, empleándose por ello fórmulas y dejándose de lado cualquier ínfula de crear un producto personal: "¡Ibáñez, usted produzca, produzca, que se vende todo!". ${ }^{40}$ Por su parte, las editoriales lanzaban sus publicaciones de forma semanal, y un retraso, aunque solo fuese de unos pocos días, amenazaba con hacer que la tirada se perdiese y descuadrase los ingresos de la empresa.

Pese a todo, el régimen seguía teniendo un control limitado de los contenidos, pues como ya hemos visto los encargados de la censura despachaban con cierta urgencia las publicaciones infantiles y juveniles, fijándose más en los aspectos gráficos, y más concretamente en los elementos eróticos, que en el mensaje de las historietas. Es por ello que ya en los años 60, desde el Ministerio de Información y Turismo se va a perfeccionar el sistema de control en el que ya se había trabajado durante la década anterior, haciéndolo mucho más efectivo.

\section{EL PERFECCIONAMIENTO DEL SISTEMA DE CONTROL (1962-1977)}

Para remediar la escasa efectividad de la legislación aprobada en la década anterior, el Ministerio de Información y Turismo realiza durante los años 60 una serie de cambios con la intención de perfeccionar el sistema de control. El primero de estos cambios va a ser poner fin a la JAPI, sustituyéndola en 1962 por una comisión asesora más amplia, la Comisión de Información y Publicaciones Infantiles y Juveniles (CIPIJ), en la que esta vez van a imperar los sectores católicos en detrimento de la Falange: los veintidós miembros de la CIPIJ se distribuyen de la siguiente manera: ocho representantes de grupos católicos, concretamente, la Comisión Católica Española de la Infancia, Gabinete Santa Teresa de Acción Católica, Asociación Católica Nacional de Padres de Familia y Comisión Episcopal de Prensa e Información, frente a seis de la Falange, ${ }^{41}$ de entre los cuales hay que contar dos representantes de la Sección

40. Ibáñez, entrevistado en El Mundo en 2017.

41. España, Orden de 13 de octubre de 1962 por la que se regula la composición y funcionamiento del Consejo Nacional de Prensa, Boletín Oficial del Estado 254, 23 de octubre de 1962, art. 6, y España, Orden de 31 de octubre de 1962 por la que se amplía la composición de la Comisión de Información y Publicaciones Infantiles y Juveniles del Consejo Nacional de Prensa, Boletín Oficial del Estado 267, 7 de noviembre de 1962. 
Femenina, donde los elementos católicos tienen un peso mayor que los fascistas. ${ }^{42}$ Además, la comisión incluía a seis vocales y el secretario, entre los que encontramos religiosos.$^{43}$ Es por ello que los sectores católicos van a tener un gran peso, que no van conservar hasta la constitución de la última Comisión en $1976 .{ }^{44}$

Sin embargo, la CIPIJ no empieza a funcionar de forma efectiva hasta el nombramiento de un secretario general, el padre dominico Jesús María Vázquez, a finales de $1963 .{ }^{45}$ Su papel en la comisión va a ser clave, consiguiendo que el Ministerio de Información y Turismo contrate a tres personas especializadas en publicaciones infantiles y juveniles, cada una de ellas situada en uno de los principales centros de publicación del país: Madrid, Barcelona y Valencia, logrando de este modo que a partir de ese momento se aplique la normativa de forma efectiva, en ocasiones incluso radical. ${ }^{46}$

Para comprender el papel de la censura con respecto al cómic femenino debemos comprender en primer lugar que el padre Vázquez se muestra como un firme defensor no solo de la normativa, sino también de la segregación por sexos en lo que a lecturas se refiere. ${ }^{47}$ Considera más que adecuada dicha segregación porque "es indudable que la evolución educativa de las niñas ha de ser, en conjunto, diferente de la de los niños [...]. Y la diferencia fundamental está en los diversos fines para que niño y niña han nacido". ${ }^{48}$ Existe por lo tanto una clara defensa del discurso tradicional que considera innato el papel que mujeres y hombres desempeñan en la sociedad: el adolescente posee una necesidad "de excitación y agitación. El niño ha salido de la heroicidad [...]. Y en todas sus lecturas busca ejemplos de hombres cuyas vidas y profesiones satisfagan sus ansias de ser grande". Por el contrario, la joven adolescente "siente una fuerte atracción por todo aquello que le enseña a ser una buena madre y ama de casa. Es su principal, y en la mayoría de los casos, única vocación humana". ${ }^{49}$ Puesto que el padre Vázquez será quien dirija al nuevo personal encargado de la censura, es indudable que esta forma de pensar va a tener un peso importante en el criterio de la censura.

42. Danièle Bussy Genevois, "Mujeres de España: de la república al franquismo", en Historia de las mujeres: el siglo XX, ed. Georges Duby y Michelle Perrot, vol. 5 (Madrid: Taurus Ediciones, 1993), 216-7.

43. España, Orden de 4 de mayo de 1966 por la que se dispone la renovación de los miembros de la Comisión de Información y Publicaciones Infantiles y Juveniles, Boletín Oficial del Estado 123, 24 de mayo de 1966, art. 2.

44. España, Orden de 31 de mayo de 1976 por la que se dispone la renovación de la Comisión de Información y Publicaciones Infantiles y Juveniles, Boletín Oficial del Estado 176, 23 de julio de 1976.

45. España, Orden de 30 de septiembre de 1963 por la que se amplía el Reglamento de la Comisión de Información y Publicaciones Infantiles y Juveniles del Consejo Nacional de Prensa, Boletín Oficial del Estado 271, 12 de noviembre de 1963, art. 6.

46. Antonio Martín, entrevistado por los autores.

47. José María Vázquez, La prensa infantil en España (Madrid: Doncel, 1963), 95.

48. Ibíd., 118.

49. Ibíd., 119-20. 
Este deseo de controlar las publicaciones infantiles y juveniles no desaparece ni mucho menos con la Ley de Prensa en marzo de 1966, pues si bien es cierto que con esta norma se acaba el control previo de la prensa, va a existir una norma especial que continuará controlando las publicaciones infantiles y juveniles..$^{50}$ Esta nueva normativa mantiene la revisión previa, realizada por la Dirección General de Prensa. ${ }^{51}$ Esto no va a cambiar hasta la llegada de las primeras reformas democráticas en 1977, muerto ya el dictador, que garantizarán la libertad de prensa y, por lo tanto, el fin de la censura. ${ }^{52}$

Tampoco varía en 1966 la esencia de la normativa, pues en la práctica la dictadura y la Iglesia se niegan a abandonar el discurso femenino de los años previos, incluso cuando la incorporación de las mujeres al mundo laboral remunerado sea un hecho normalizado: se justifica entonces que sus papeles son aceptables porque trabajan subordinadas a los hombres, y se espera que sus jornadas laborales no afecten en modo alguno a la atención que presentan a sus familias. ${ }^{53}$ Por lo tanto, la nueva normativa para publicaciones infantiles y juveniles cambia el lenguaje, pero no la esencia: encontramos que no se pueden mostrar ni conductas ni hechos que puedan ser tachados de inmorales, escenas que posean algún tipo de erotismo, se burlen de la tradición y costumbres españolas, o fomenten el culto desproporcionado a uno mismo, igual que queda prohibida toda presentación de asuntos que no se consideren adecuados para el público infantil. ${ }^{54}$

Ahora bien, nuevamente nos encontramos con una normativa muy general, por lo que la clave para conocer su impacto está en la forma en que dichas normas se interpretan. $\mathrm{Y}$ es que un amor ilegítimo puede interpretarse de muy amplia manera, desde una relación con una persona casada, pasando por una pareja en la que uno de los dos no tenga intenciones de casarse, e incluso una relación normal pero que no cuente con el beneplácito de los padres de alguno de los enamorados. De igual modo, el respeto a la tradición y la forma de vida española puede depender de la perspectiva: ¿se refiere la norma a la forma de vida del censor, una persona adulta que ha vivido la rígida mo-

50. España, Ley 14/1966, de 18 de marzo, de prensa e imprenta, Boletín Oficial del Estado 67, 19 de marzo de 1966, art. 15.

51. España, Decreto 195/1967, de 19 de enero, por el que se aprueba el Estatuto de Publicaciones Infantiles y Juveniles, Boletín Oficial del Estado 37, 13 de febrero de 1967, art. 22.

52. España, Real Decreto Ley 24/1977, de 1 de abril, sobre libertad de expresión, Boletín Oficial del Estado 87, 12 de abril de 1977.

53. María del Carmen García-Nieto París, "Modelo de mujer que creó el franquismo y los colectivos de mujeres que generó", en Historia de las mujeres: el siglo XX, 663.

54. España, 1967, arts. 8-9. 
ral de la posguerra, o a la de las lectoras, criadas en una España en la que el consumo ya se ha convertido en una forma de vida?

Los testimonios de algunas autoras de la época muestran que a partir de este momento la censura no se aplica ya solamente sobre los dibujos, sino que el nuevo personal censor se toma tiempo en leer las historias: "La censura se metió con [el personaje de la periodista] Mary Noticias [...] Dijeron: 'Esta mujer es muy libre. ¿No tiene familia?' Tuvimos que representar que era Navidad, y que Mary iba a su casa". ${ }^{55} \mathrm{Y}$ puesto que muchas de estas publicaciones infantiles y juveniles poseen una periodicidad semanal, las editoriales son las primeras que no quieren tener problemas con la censura, pues eso significa inevitablemente salir al mercado con retraso, mientras que el personal creativo no desea tener que reescribir o dibujar de nuevo una escena, pues ese trabajo no se cobra.

La práctica de la autocensura la confirma el hecho de que Editorial Bruguera, la principal editora de la época, difunde en 1964 una serie de normas internas más estrictas que las de la propia censura, de tal modo que el trabajo de sus guionistas y dibujantes no encuentre reticencias. ${ }^{56} \mathrm{El}$ personal creativo de las editoriales reconoce que hay cosas que simplemente es mejor no mostrar, aunque no se recoja en la ley: "No podías dibujar un hombre y una mujer solos, siempre tenías que poner otra persona detrás [...] Y, eso sí, la historia siempre tenía que acabar en boda" ${ }^{57}$ Hay, por lo tanto, una aceptación absoluta de la normativa censora, sintiendo que no pueden hacer nada al respecto: "Eran tiempos difíciles, no podías levantar muy alto la voz; se veía como si tuvieras un corsé que te estuviera oprimiendo, veías o sentías que había censura, que había cosas que no se podían decir o contar, y cada uno intentaba vivir lo mejor que podía". ${ }^{58}$

Esta aceptación por parte de editoriales y personal artístico nos puede hacer pensar que la censura era imbatible en la España de Franco, si bien nada más lejos de la verdad. La Ley de Prensa de 1966 permitía ciertos espacios para la resistencia, que si bien eran pocos, indudablemente ofrecían una mayor libertad que en décadas anteriores. ${ }^{59}$ Muestra de ello es que algunas publicaciones periódicas para adultos, como las revistas Cuadernos para el Diálogo o Cambio 16 sufrían constantes sanciones, pero sus

55. Carme Barberà, entrevistada en El Periódico en 2014, 〈http://www.elperiodico.com/es/sociedad/20140424/ carme-barberahasta-la-censura-se-metio-con-mary-noticias-3257260), consulta 22 de julio de 2017.

56. Vicent Sanchis, Tebeos mutilados (Barcelona: Ediciones B, 2010), 115.

57. Trinidad Tinturé, entrevistada en ForoEsther n. $^{\circ} 37$ (2013): 16.

58. Carlos Pino, entrevistado en Tebeosfera en 2003, 〈http://www.tebeosfera.com/1/Documento/Entrevista/ Pino/Carlos.htm>, consulta 6 de diciembre de 2014.

59. España, Ley 14/1966, de 18 de marzo, de prensa e imprenta, Boletín Oficial del Estado 67, 19 de marzo de 1966. 
tiradas crecieron (multiplicándose por diez en algunos casos) justamente por ofrecer contenidos más acordes a los intereses de la población, haciendo que el riesgo de las multas valiese la pena.

Cierto es que en la prensa para adultos se había puesto fin a la censura previa, cosa que no ocurrió con las publicaciones infantiles y juveniles, pero igualmente existían herramientas legales para enfrentarse a las decisiones de la CIPIJ, que hasta donde sabemos nunca fueron explotadas por las editoriales. Efectivamente, el miedo de no salir a tiempo a los puntos de venta seguía estando presente, pero sin duda también existió cierto conformismo que hizo que, al menos las principales editoriales, no presionaran para favorecer cambios. Es por ello que el historiador Antonio Martín sentencia:

Soy del parecer de que las empresas editoriales españolas de tebeos también fueron responsables, en gran medida, de la existencia, potencial dureza y duración de la censura previa de las publicaciones infantiles y juveniles [...]. Es un hecho que estas empresas -al menos las de mayor tamaño y envergadura económica- podían haber puesto en juego a sus abogados para recurrir las decisiones de los censores o, como mínimo, para intentar "negociar" de manera efectiva con la CIPIJ. Y todo ello sin contar con posiblemente los mecanismos legales que daban soporte a dicha Comisión se podrían haber analizado y cuestionado desde el mismo corpus jurídico del régimen. ${ }^{60}$

\section{CONCLUSIONES}

La censura a las publicaciones infantiles y juveniles durante el franquismo tiene un objetivo fundamental: controlar el contenido de las revistas destinadas a las y los menores para convertirlas en un vehículo de transmisión de los valores que propugnaba la dictadura. En el caso de las publicaciones femeninas, el objetivo es reforzar los roles de madre y esposa, la moral cristina y el atractivo del espacio doméstico.

Este control es especialmente efectivo en los años posteriores a la Guerra Civil, cuando el número limitado de revistas permite la supervisión de los directores de las publicaciones, aunque el aumento del número de revistas desde finales de los años 40 obligará a diseñar una estructura de control más compleja: un comité especializado afín a la dictadura que asesore a las autoridades, además de un código de conducta que fija líneas rojas que no se pueden traspasar. Sin embargo, al no crear estructuras de comunicación, ni dotar de medios a dicho comité, los censores del régimen prestan poca

60. Antonio Martín, entrevista realizada por el autor vía email entre los días 21 y 25 de julio de 2017. 
atención a las publicaciones infantiles y juveniles, centrándose principalmente en los aspectos gráficos, sobre todo en evitar elementos que puedan considerarse eróticos.

Consciente de que el sistema de control ideado durante la década de los 50 no estaba funcionando, el Ministerio de Información y Turismo comenzó a reaccionar a partir de 1962, creando una comisión asesora que se organiza de manera más efectiva, abriendo puestos específicos para censores de publicaciones infantiles y juveniles, además de desarrollar un nuevo cuerpo normativo. Todo ello coincide en el tiempo con la modernización del país y la aparición de nuevos valores entre la juventud, algo que sin duda debe de ser considerado especialmente pernicioso en el caso de las niñas y adolescentes, que pueden sentirse más interesadas por incorporarse al mundo del trabajo remunerado y optar por una vida más independiente antes que aceptar un papel meramente doméstico.

Posiblemente por ello, por combatir contra unos valores cambiantes, la normativa franquista se muestra vaga, dejando mucha libertad al criterio subjetivo de los censores. De este modo, editoriales, guionistas y dibujantes se ven impelidos a crear historias pensando ya de antemano en lo que el censor considerará apropiado, aplicando una autocensura que va más allá de los aspectos contemplados explícitamente en la legislación. De esta manera no solo se evita que se traten temas que la dictadura considera delicados (el divorcio, el adulterio, el deseo, etc.), sino que se crean relatos que casan con los ideales del régimen (amores idealizados, noviazgos destinados al matrimonio, mujeres que trabajan, pero anteponen siempre su familia a sus carreras, etc.).

De este modo, el sistema de control ideado por el franquismo resulta doblemente útil: no solo evita que se publiquen cómics que puedan lanzar mensajes contrarios a los del régimen, sino que además logra que las propias editoriales y su personal artístico produzcan obras más conservadoras de las que posiblemente, de haberse dado otras condiciones, habrían realizado. Las condiciones del sistema editorial van a reforzar esta cooperación: tanto las editoriales como su personal sienten una presión económica, a la par que no tienen la sensación de estar produciendo una obra artística, por lo que aceptan en la práctica las limitaciones que se les marcan.

\section{BIBLIOGRAFÍA}

Barrero, Manuel. "Nueva mirada sobre la producción editorial de tebeos durante los años cuarenta", Espacio, tiempo y forma. Serie V, n. ${ }^{\circ} 26$ (2014): 89-113.

Blasco Herranz, Inmaculada. "Más poderoso que el amor: género, familia, piedad y política en el movimiento católico español”. Pasado y Memoria. Revista de Historia Contemporánea, n. ${ }^{\circ} 7$ (2008): 79-100. 
Bussy Genevois, Danièle. "Mujeres de España: de la república al franquismo". En Historia de las mujeres: El siglo XX, editado por Georges Duby y Michelle Perrot. Vol. 5, 203-22. Madrid: Taurus, 1993.

Domínguez Prats, Pilar, y María del Carmen García-Nieto París. "Franquismo: represión y letargo de la conciencia feminista, 1939-1977”. En Historia de las mujeres. Una historia propia, editado por Bonnie S. Anderson y Judith P. Zinsser. Vol. 2, 640-8. Barcelona: Crítica, 1991.

Folguera Crespo, Pilar. "El Franquismo. El retorno a la esfera privada (1939-1975)". En Historia de las Mujeres en España, editado por Elisa Garrido, 527-48. Madrid: Síntesis, 1997.

Fusi, Juan P. “La cultura”. En Franquismo: El juicio de la historia, coordinado por J. L. García Delgado, 219-96. Madrid: Temas de Hoy, 2005.

García-Nieto París, María del Carmen. "Modelo de mujer que creó el franquismo y los colectivos de mujeres que generó". En Historia de las mujeres: El siglo XX, editado por Georges Duby y Michelle Perrot. Vol. 5, 640-68. Madrid: Taurus, 1993.

Gil Roësset, Consuelo. La Pedagogía en la prensa infantil: conferencia pronunciada por Consuelo Gil Roësset de Franco el día 22 de mayo de 1947. Madrid: Escuela Social de Madrid, 1947.

Gubern, Román. La censura. Función política y ordenamiento jurídico bajo el franquismo (1936-1975). Barcelona: Península, 1981.

Jiménez Morales, Rosario. "Pequeños defectos que debemos corregir: aprendiendo a ser mujer en la historieta sentimental de los años cincuenta y sesenta". ARBOR. Vol. CLXXXVII, extra 2 (2011): 159-68.

Juliá, Santos. "La sociedad”. En Franquismo: El juicio de la historia, coordinado por J. L. García Delgado. Madrid: Temas de Hoy, 2005.

Loranca de Castro, María del Pilar. "Mis Chicas y su influencia en las niñas de posguerras", Historietas, n. ${ }^{\circ} 3$ (2013): 71-81.

Marshment, Margaret. "The Picture is Political: Representation of Women in Contemporary Popular Culture". En Introducing Women's Studies, editado por Victoria Robinson y Diane Richardson, 125-51. Londres: MacMillan Press, 1997.

Martín, Antonio. Apuntes para una historia de los tebeos. Barcelona: Glénat, 2000.

Moix, Terenci. Historia social del cómic. Barcelona: Ediciones B, 2007 [1968].

Morcillo Gómez, Aurora. En cuerpo y alma. Ser mujer en tiempos de Franco. Madrid: Siglo XXI, 2015.

Pérez de Urbel, Justo. "Las revistas infantiles y su poder educador”. Revista Nacional de Educación $\mathrm{n}^{\circ} 1$ (1941): 55-8.

Preston, Paul. Franco. Caudillo de España. Barcelona: DeBolsillo, 2002.

Ramírez, Juan. El “comic” femenino en España. Arte sub y anulación. Madrid: Cuadernos para el Diálogo, 1975. 
Rodríguez Moreno, José J. “La imposición de los valores católicos patriarcales a través de la censura en las revistas juveniles femeninas de la España franquista (1941-1977)". Feminismo/s n. ${ }^{\circ} 28$ (2016): 235-68.

Sánchez Jiménez, José. “La jerarquía eclesiástica y el Estado franquista: las prestaciones mutuas". Ayer 33 (1999): 167-86.

Sanchis, Vicent. Tebeos mutilados. Barcelona: Ediciones B, 2010.

Valencia Fernández, Celia. "Las políticas para las mujeres trabajadoras durante el franquismo". En Mujeres y hombres en la España franquista: Sociedad, economía, política, cultura, editado por Gloria Nielfa Cristobal, 145-78. Madrid: Editorial Complutense, 2003.

Vázquez, José María. La prensa infantil en España. Madrid: Doncel, 1963.

\section{EnTrevistas}

Barberà, Carme. Entrevistada en El Periódico en 2014. 〈http://www.elperiodico.com/es/so ciedad/20140424/carme-barberahasta-la-censura-se-metio-con-mary-noticias-3257260 . Consulta 22 de julio de 2017.

Domínguez Navarro, Manel. Entrevistado en Tebeosfera en 2010. 〈http://www.tebeosfera.com/ documentos/textos/manel_dominguez_navarro_entrevista.html〉. Consulta 9 de marzo de 2016.

Ibáñez, Francisco. Entrevistado en El Mundo en 2017. 〈https://www.elmundo.es/papel/cultura /2017/11/17/5a0db5bfe2704ea07e8b45ad.html>. Consulta 10 de mayo de 2019.

Martín, Antonio. Entrevista realizada por el autor vía email entre los días 21 y 25 de julio de 2017.

Pino, Carlos. Entrevistado en Tebeosfera en 2003. 〈http://www.tebeosfera.com/1/Documento/ Entrevista/Pino/Carlos.htm>. Consulta 6 de diciembre de 2014.

Tinturé, Trinidad. Entrevistada en ForoEsther, n. ${ }^{\circ} 37$ (2013): 13-8.

\section{LEGISLACión}

España. Ley de prensa, de 23 de abril. Boletín Oficial del Estado 550, 24 de abril de 1938.

-. Orden de 21 de enero de 1952 por la que se crea la Junta Asesora de la Prensa Infantil. Boletín Oficial del Estado 21, 1 de febrero de 1952.

-. Orden de 5 de febrero 1952 por la que se crea la Junta Asesora de la Prensa Infantil. Boletín Oficial del Estado 52, 21 de febrero de 1952.

- Decreto de 24 de junio de 1955, por el que se establecen las normas a que han de ajustarse las publicaciones infantiles y juveniles. Boletín Oficial del Estado 204, 23 de julio de 1955.

-. Orden de 24 de junio de 1955, por la que se desarrolla el Decreto sobre ordenación de las publicaciones infantiles y juveniles. Boletín Oficial del Estado 33, 2 de febrero de 1956. 
- Decreto 2551/1962 de 27 de septiembre, por el que se reorganiza la Dirección General de Prensa. Boletín Oficial del Estado 245, 12 de octubre de 1962.

- Orden de 13 de octubre de 1962 por la que se regula la composición y funcionamiento del Consejo Nacional de Prensa. Boletín Oficial del Estado 254, 23 de octubre de 1962.

_. Orden de 31 de octubre de 1962 por la que se amplía la composición de la Comisión de Información y Publicaciones Infantiles y Juveniles del Consejo Nacional de Prensa. Boletín Oficial del Estado 267, 7 de noviembre de 1962.

_. Orden de 30 de septiembre de 1963 por la que se amplía el Reglamento de la Comisión de Información y Publicaciones Infantiles y Juveniles del consejo Nacional de Prensa. Boletín Oficial del Estado 271, 12 de noviembre de 1963.

- Orden de 4 de mayo de 1966 por la que se dispone la renovación de los miembros de la Comisión de Información y Publicaciones Infantiles y Juveniles. Boletín Oficial del Estado 123, 24 de mayo de 1966.

- Ley 14/1966, de 18 de marzo, de prensa e imprenta. Boletín Oficial del Estado 67, 19 de marzo de 1966.

- Decreto 195/1967, de 19 de enero, por el que se aprueba el Estatuto de Publicaciones Infantiles y Juveniles. Boletín Oficial del Estado 37, 13 de febrero de 1967.

- Orden de 31 de mayo de 1976 por la que se dispone la renovación de la Comisión de Información y Publicaciones Infantiles y Juveniles. Boletín Oficial del Estado 176, 23 de julio de 1976.

- Real Decreto Ley 24/1977, de 1 de abril, sobre libertad de expresión. Boletín Oficial del Estado 87, 12 de abril de 1977. 


\title{
ANEXO: CÓMICS FEMENINOS DE LA DICTADURA
}

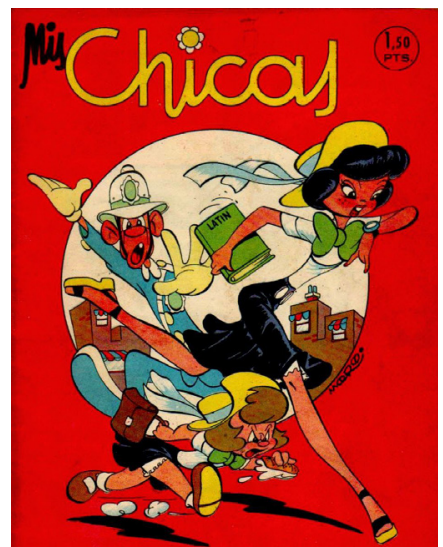

\author{
Ilustración 1. Portada de Mis Chicas
}

La revista femenina pionera, Mis Chicas, muestra un modelo ideal de mujer: hermosa y elegante, pero al mismo refleja los tópicos tradicionales, como su incapacidad para llegar a tiempo o su escasa habilidad para manejarse fuera del hogar, como muestra el rostro enfurecido del agente de tráfico que las ve pasar sin mirar por dónde avanzan; la revista fue uno de los referentes de la dictadura en cuanto a publicaciones infantiles y juveniles. Portada de Mis Chicas n. 382 (1949), editorial Chicos.

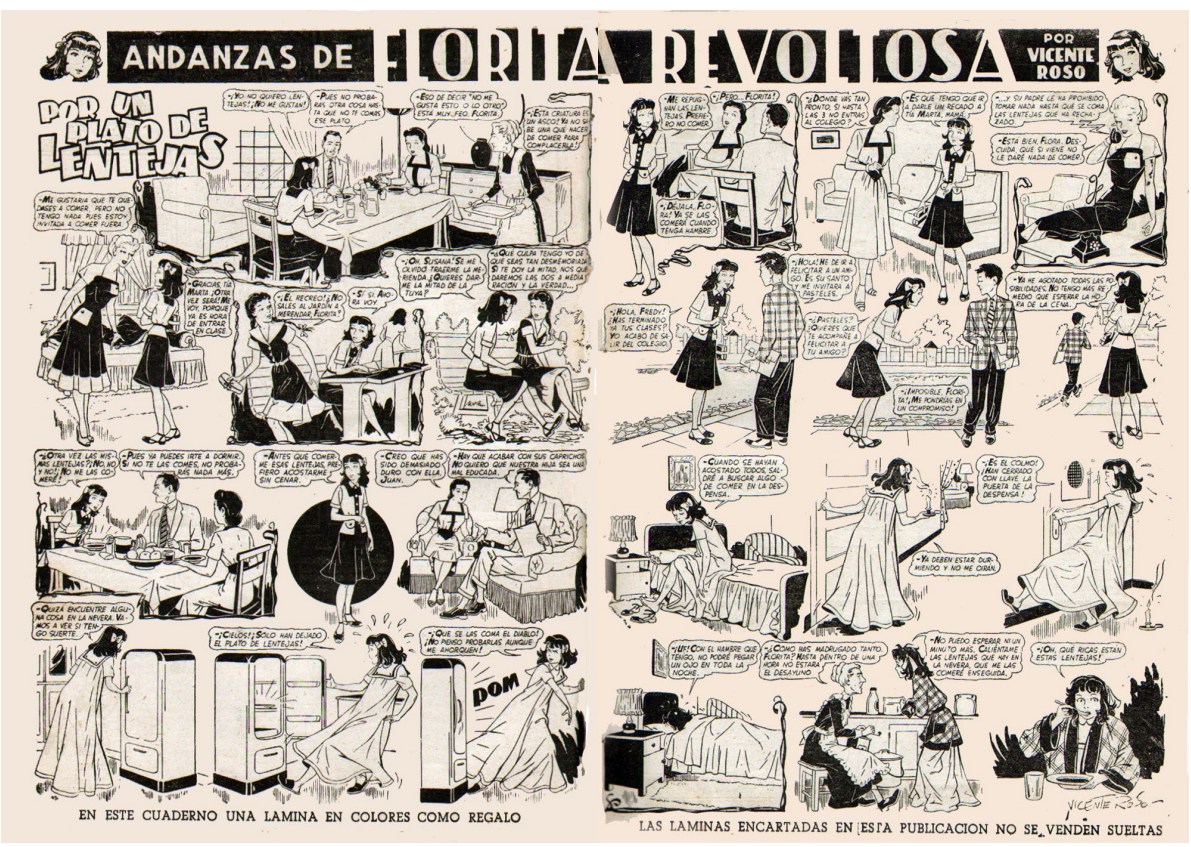

Un ejemplo de tebeo femenino publicado por Clíper, una editorial sin vínculos directos con el régimen. La comicidad de la historia parte del hecho de que Florita se muestra caprichosa con la comida, ante lo cual el padre actúa con su autoridad de cabeza de familia, prohibiendo a su hija comer otra cosa hasta que despache el plato de lentejas que ha dejado; tras varias peripecias, la muchacha se come las lentejas encantada, mostrándose de ese modo la sabiduría del patriarca; mediante el humor, la publicación reforzaba los valores tradicionales de autoridad y jerarquía. Páginas interiores de Florita n. 2 (1949), editorial Clíper. 


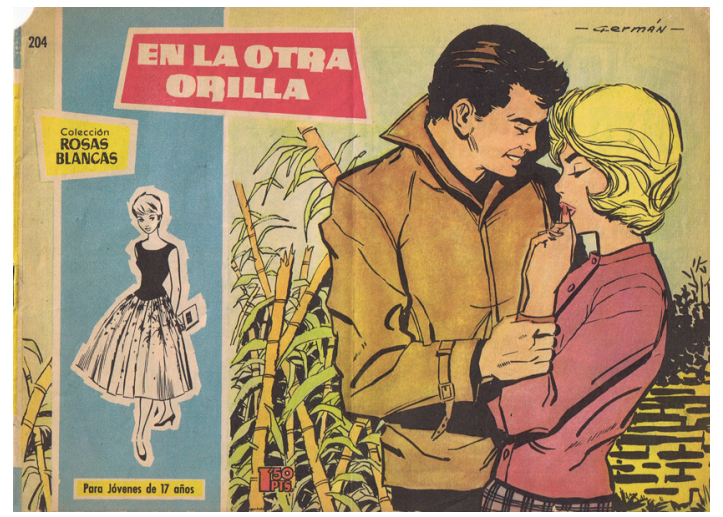

\section{Ilustración 3. Portada de Rosas Blancas}

El amor se convirtió en un elemento común de los tebeos femeninos, mostrándose en sus páginas cuáles eran las conductas adecuadas para tener éxito en una relación, siempre desde un punto de vista idealizado y, por supuesto, totalmente asexuado que presentaba el amor como un sentimiento que nada tenía que ver con la pasión física. Portada de Rosas Blancas $n .^{\circ} 198$ (1958), editorial Toray.

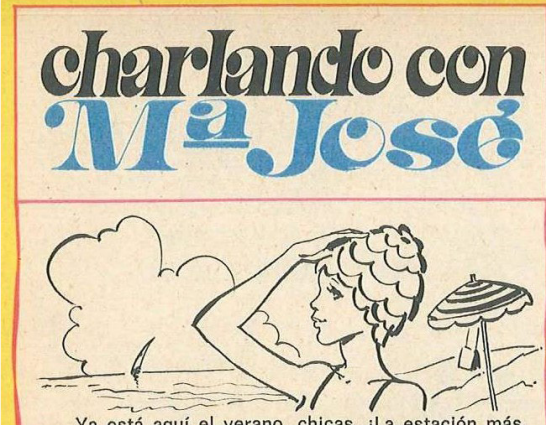

Ya está aquí el verano, chicas. iLa estación más feliz del año! Con los primeros baños de sol os ha béis puesto más guapas. Pero cuidado: mientras se béis puesto más guapas. Pero cuidado: mientras Resulta fatal secarlo al sol después del baño. Así que, al equiparos para el verano, no os contentéis con compraros un bañador muy "in". Pensad también en un gorro de baño para defender vuestro cabello, que ahora hay que cuidar más que nunca.

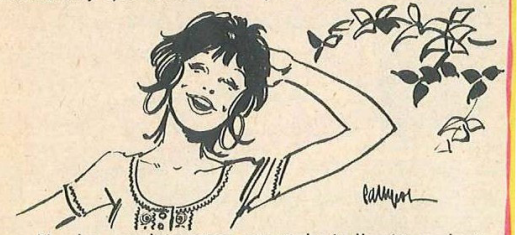

Y a las recalcitrantes que todavía lleváis melenita, os diré que estáis muy anticuadas... ¡Pero si hasta Los Beatles han renunciado a ella! Adelante, rezagadas. Se impone un buen corte de pelo para que éste soporte cómodamente las tiranías a que lo sometéis durante el calor.

Claro que los cuidados que debéis a vuestro cabello no disminuyen con su medida. Lavadlo como mi nimo una vez por semana, con el champú adecuado y cepilladlo todas las noches. ¡No pongáis el grito en el cielo! No tienen que ser cien veces. Mejor pocas y todos los días. Es el sistema ideal para librarlo del polvo y dejarlo suelto y brillante.

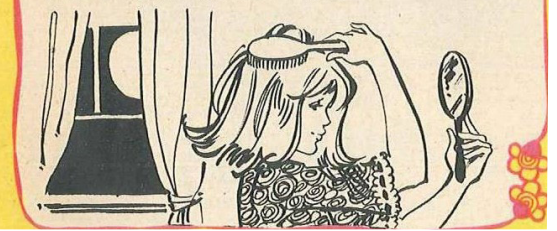

\section{Ilustración 4. Portada de Claro de Luna}

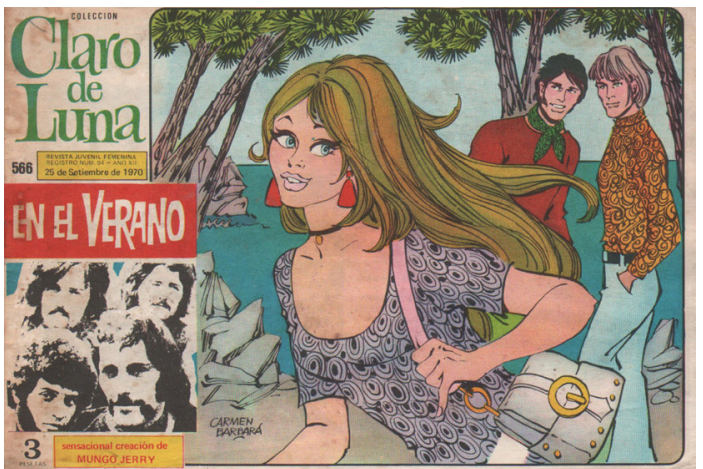

El desarrollo económico del país y la aparición de una cultura juvenil inspiró publicaciones como Claro de Luna, en las cuales se adaptaban canciones de moda para crear relatos románticos. Pese a la supuesta carga de modernidad de las historietas, era evidente que la censura controlaba los contenidos, como muestra el amplio escote sin curvas que luce la protagonista. Portada de Claro de Luna n. ${ }^{\circ} 566$ (1970), editorial Ibero Mundial de Ediciones.

\section{Ilustración 5. Interior de Lily}

La supuesta modernidad de los años setenta era más superficial que auténtica. La sección de consejos femeninos de Lily seguía centrándose en aspectos superficiales como la moda y la belleza, convirtiendo las revistas para niñas y adolescentes en un mero puente hacia las revistas femeninas. Interior de Lily n. ${ }^{\circ} 444$ (1970), editorial Bruguera. 